II.

\title{
Über das Verhalten der Sensibilität bei Hirnrindenläsionen.
}

Von

\section{Prof. Dr. K, Bonhoeffer (Königsberg i. Pr.).}

(Mit 1 Fig. im Text.)

Von den Ausfällserscheinungen, welche durch isolierte Läsionen im Bereiche der kortikalen Armregionen verursacht werden, sind die motorischen in der Hauptsache klargestellt. In allen Fällen findet sich als definitive Ausfallserscheinung eine vor allem die feineren $\mathrm{Be}$ wegungen der Hand, die Handfertigkeiten betreffende Störung, während die groben motorischen Leistungen eine nur geringe, oft gar keine Schädigung aufweisen. Eine kortikale monoplegische Störung im Armgebiet derart, dass sie etwa nur den Schultergürtel oder die Bewegungen im Ellbogengelenk beträfen und die Hand frei liessen, wird nie angetroffen. Es kann deshalb von einer gliedweisen, den Gelenkabschnitten entsprechenden Projektion der Motilität in der Hirnrinde des Menschen entsprechend den Munkschen Anschauungen nach den Erfahrungen der Klinik nicht eigentlich gesprochen werden. Stets ist vor allem die Hand geschädigt und es ist dies auch begreiflich, da die Hand, dieses beim Menschen funktionell so eminent fein differenzierte Organ, anch die stärkste und ausgedehnteste Rindenvertretung besitzen wird.

In der Frage nach den sensiblen Funktionen der Armregion und überhaupt der motorischen Zone ist dagegen noch manches strittig. Nach Munk sind die motorisch erregbaren Zonen gleichzeitig die sensiblen Rindenfelder für den betreffenden Gliedabschnitt. v. Monakow betrachtet die motorischen Rindenfelder zwar als ein Teilgebiet der Fühlsphäre, diese überragt aber nach ih $\mathrm{m}$ die motorische Zone lateral-, occiptal- und medialwärts. Redlich u. A. erblicken dagegen im Parietalhirn ein Zentrum der Hautsensibilität und des Muskelsinns.

Ein neuer Gesichtspunkt ist von Wernicke in die Frage nach den sensiblen Funktionen der motorisehen Rindenfelder gebracht worden an der Hand zweier Beobachtungen von Rindenläsion im mittleren Drittel der Zentralwindungen, bei denen er einen Verlust der Fähigkeit, Gegenstände durch Tasten wiederzuerkennen, nachwies. Das Neue war, dass er den Hauptnachdruck auf die Feststellung legte, dass bei dieser sogenannten Tastlähmung die begleitende Sensibilitätsstörung so gering 
war, dass sie eine Erklärung für den Verlust des taktilen Wiedererkennens nicht abgab. Er schliesst aus diesen Befunden, dass die Armregion, speziell ihr hinterer Teil, die Tastrorstellungen beherberge, und stellt dieses Territorium funktionell in eine linie mit den Rindengebieten der motorischen und sensorischen Aphasie. Diese Tastlähmung: ist nach Wernicke ein differentiell-diagnostisch wichtiges Rindensymptom. Anders äussert sich D ejerine. Der sogenannte stereognostische Sinn hat nach ihm keine lokalisatorische Bedeutung. Man finde vielmebr bei Tastlähmung immer mehr oder weniger ausgesprochene Sensibilitätsstörungen, die bald mehr die tiefere, bald mehr die oberflächliche Sensibilität betreffen. Die Differentialdiagnose zwischen kortikaler und Kapselhemianästhesie könne nicht durch den Charakter der Hemianästhesie entschieden werden. v. Monakow nähert sich der Wernickeschen Lehre. Er hält Defekte der Stereognose, allerdings auch des Muskelsinns, bei gut erhaltener Berührungs- und Schmerzempfindung für wichtig zur Diagnose einer Rindenläsion.

Neuere Beobachter schliessen sich bald mehr Wernicke an (Walton und Paul, Williamson), andere an v. Monakow (Diller).

Bei dieser Sachlage mag es gestattet sein, nene Beobachtungen über partielle Rindenläsionen im Bereiche der Armregion bekannt zu geben mit besonderer Berücksichtigung der Sensibilitätsverhältnisse.

Um Wiederholungen zu vermeiden, habe ich die Protokolle, die sich speziell mit den sensiblen Verhältnissen und dem taktilen Wiedererkennen beschäftigten, nur im ersten Falle ausführlicher wiedergegeben. Auch im übrigen habe ich mich bei Wiedergabe der Krankengeschichten auf die hier interessierenden Daten beschränkt.

\section{Beobachtung I.}

Pialäsion im Bereich der mittleren Zentralwindung. Leichte motorische Störungen der linken Hand. Störung der Tastfähigkeit und der Lokalisation.

R., 24 Jahre alt, leidet seit dem 12. Lebensjahre an Krämpfen, hat das Gymnasium bis Untersekunda besucht. Die epileptischen Anfälle sind sehr häufig, meist nachts. Entwicklung epileptischer Reizbarkeit.

Im Jahre 1900 wurde eine rechtsseitige osteoplastische Schädelresektion gemacht, dabei die Unterbindung einiger stark gefültter Piavenen im Bereich des mittleren Drittels der vorderen Zentralwindung vorgenommen, in der Erwartung, dadurch vielleicht eine günstige Beeinflussung der Epilepsie zu erzielen.

Es tritt gleich nach der Operation Unsicherheit in der linken Hand ein; R. merkte, dass er im Greifen ungeschickter war, er spielte am Klavier die Begleitung schlechter.

Die objektive Untersuchung ergab einige Monate nach der Operation, dass feinere Fingerbewegungen links schlechter ausgeführt wurden als rechts. Die Opposition des Daumens war ungestört, überhaupt waren alle 
Bewegungen möglich, subjeltiy hatte er aber ein Gefühl der Steifigkeit und Ungeschicklichkeit. Eine Veränderung der passiven Beweglichkeit bestand nicht. Die grobe Kraft war, dynamometrisch gemessen, rechts und linlks $(34: 32)$, entsprechend dem physiologischen Verbältnis. Eine Sensisibilitätsprüfung ergab gut erbaltene Schmerz-, Temperatur- und Berührungsempfindung, passive Bewegungen in den einzelnen Fingergelenken wurden wahrgenommen und die Richtang der Bewegung angegeben. Die Lokalisation der Bertihrungen geschah rechts ganz gut und scharf an der ganzen Hand, links ist die Lokalisation im Handteller und am 4. und 5. Finger auch gut. Am Zeigefinger wird in der zweiten und Endphalanx schlecht lokalisiert, er bezeichnet den Handteller als Ort der Berührung. Die Berührung der Kuppe des Zeigefingers, des Daumens und des Mittelfingers wird sehr ungenau lokalisiert und vielfach der falsche Finger als Ort der Berührung benannt. Die Resultate werden nach kurzer Prüfung rasch schlechter, rechts bleiben sie gut.

Ich lasse einige im Laufe der letzten 2 Jahre aufgenommenen Protokolle über das taktile Erkennen bei geschlossenen Augen folgen:

Januar 1901: Schere? nicht erkannt.

Bleifeder? nicht.

Streichholz? Was geringes, ein Stäbchen, Schnur.

Wolle? lederartig.

Karton? Pappe.

Pappschachtel? Von Pappe etwas zus ammengebogenes.

Stahlfeder? 1 Stückchen Streichholz, Feder vielleicht.

Messer? Schlüssel, später Messer.

Schlüssel? Vielleicht eine Schere.

Drücker? Messer, Schlüssel.

2. II. 01. Etwas benommen.

Uhr? Schachtel, auch rechts nicht erkannt.

L. Schlüssel langsam erkannt.

9. II. Schlüssel? nicht.

Hemdknopf? Ganz klein, rund, vielleicht ein Ring.

Silberne Kugel mit Kette? glatt, vielleicht 1 Federhalter.

Radiergummi? Blöckchen.

Schraube? Dünner, weicher Gummi*) oder Bleistift lang und schmal.

Knopf? Nagel, Feder.

Karton? Stück Papier.

Später am selben Tage

Streichholzschachtel erkannt.

Kleiner Schlüssel erkannt.

Schachteldeckel? Schachtel.

Rundschriftfeder? 1 kleiner Uhrschlüssel.

Kalender? Papier.

1 M.? 1 Schlüssel.

Uhrschlüssel? Das kann 1 Uhrschlüssel, ein kleiner niedlicher sein.

Lokalisation der Berührung bei den ersten 6 Versuchen an den Fingerkuppen richtig, dann schlecht.

*) Die taktil nicht erkannten Gegenstände werden dem Kranken regelmässig nachträglich gezeigt. 
19. V. 1901. Tastprüfung.

Harnadel? Draht, Feder.

Hemdknöpfchen? Dreikantig.

Schraube? Kalt, Eisen, oben wie ein Knopf.

Dominostein? 1 Stïck Blech.

Gummi? auch ein Dominostein.

Kugel mit Kette? An einer Kette, so noch etwas.

Würfel? Der Gummi.

Schlüsselchen? Die Schraube.

10 Pfg.-Stück? Der Schlüssel.

Abgebrochener Kamm? Blechartig, 1 Stück Blech, unten so zahnartig wie bei Sägen.

Zigarrenspitzenetui? $1 \mathrm{Kamm}$ mit Zuhalter, oben stärker, so von dieser Höhe (gibt 2 fache Länge, 3 fache Breite an).

Bindfaden? Band.

Berührungsempfindung gut; Lokalisation vielfach gut, doch am Zeige-, Mittelfinger oft Fehler um ein Glied, bezeiclinet oft falsche Finger als berührt, bleibt aber meist auf derselben Tastebene; auch dorsal dasselbe Verhalten.

Lageempfindung ohne Störung.

Schwellenwertprüfung versagt.

21. I. 1908.

Lokalisation der Berührungen ungenau; bezeichnet oft falsche Finger, den Daumen anstatt des Zeigefingers, den Ringfinger anstatt des Kleinfingers. Fingerkuppenberübrung wird an das 1. Glied oder auch an die Mittelhand lokalisiert. Je länger die Untersuchung, um so schlechter die Lokalisation, dann auch rechts schlecht lokalisiert. Vereinzelt werden Berührungen genau lokalisiert. Stichförmige Berührung, starker Druck wird gut lokalisiert. Passive Bewegungen des Daumens werden richtig wahrgenommen. Am Zeigefinger werden Bewegungen im 1. Glied richtig, im 2. schwankend, im 3. noch schlechter angegeben. In den übrigen Fingern gute Lageempfindung.

Abtasten von Gegenständen: Bauer (Schachfigur)? Links: Das kann iril mir nicht enträtseln, ich halte es für ein Instrument, es war jedenfalls Holz.

Kamm? Das ist Holz, so gross wie mein Zeigefinger.

Schlüssel? Blech, auch ein Kamm.

Würfel? Schlüssel.

Wie gross? Spannt mit der rechten Hand etwa $10 \mathrm{~cm}$.

Hemdknopf? Bemerkt er überhaupt nicht.

Holzbuchstaben? 1 Stück Holz, kann aber die Form nicht beschreiben.

An diesem Tage zeigt $R$. auch bei dem Betasten mit der rechten Hand. Schwierigkeiten.

Am 24. II.

Berührungs und Schmerzempfindung, faradolsutane Sensibilität erhalten.

Lokalisation ohne Fehler im Handteller, an den Fingern oft die einzelnen Finger verwechselt, während die Querebene ungefähr getroffen ist. Die Lageempfindung an dem Handgelenk und den Endphalangen gut, Zeigefinger wie oben nicht ganz sicher.

Die Abschätzung von Gewichtsdifferenzen bei geschlossenen Augen geschieht links leidlich, während die Schätzung der Längenverhältnisse schlecht ist. 
Im 31. III.

Lokalisation der Berührungen fast gut.

Sensibilität sonst wie oben.

Tasten links:

Zündholzschachtel? Schachtel.

Knopf? nicht.

Dominostein? nicht.

Holzbuchstabe B? Erkannt.

Kamm?" nicht.

E? nicht.

In motorischer Beziehung ist noch derselbe Status wie im Jahre 1901. Es bestehen keine Spasmen. Die einzelnen feineren Fingerbewegungen geschehen etwas, aber nur wenig ungeschickter als rechts. Bei Opposition des Daumens treffen die Finger gelegentlich nicht ganz zusammen. Bei Spreizung der Finger bleiben sie nicht ganz in einer Ebene.

Die Natur der Gehirnläsion ist klar. Durch eine Unterbindung von Piavenen wird immer gleichzeitig der zugehörige, darunter liegende Rindenteil geschädigt. In der Hauptsache handelt es sich wohl um eine Kombination von kleinen durch Gefässverlegung zustande gekommenen oberflächlichen Erweichungen und von mehr oder weniger ausgedehnten, durch die Lösung der Pia bedingten Hämorrhagien in die Rinde. Das Endresultat ist eine oberflächliche Rindennarbe, im vorliegenden Falle im Bereich des mittleren Drittels der vorderen Zentralwindungen. Als Folge dieser Läsion tritt eine geringe Störung der feineren Fingerbewegungen auf, während eine Abnahme der Kraft des Händedrucks nicht statthat. Es fehlen Spasmen und Kontrakturstellung. Bei Spreizung der Finger bleiben die Finger nicht in einer Ebene.

Hinsichtlich der Sensibilität bestehen dauernde, wenn auch hinsichtlich der Intensität schwankende Ausfallserscheinungen. Die Wabrnehmung von Berührungen, von Temperatur und Schmerz ist konstant erhalten und zeigt keine Unterschiede gegenüber der gesunden Seite. Nicht so konstant verhält sich die Lokalisationsfähigkeit der Berührungen. Es kommen grobe Fehler in der Art, dass etwa Berührungen, welche die Hand treffen, an den Vorderarm lokalisiert wurden, zwar nicht vor. Aber trotzdem besteht eine deutliche Störung der Lokalisation, die um so stärker wird, je weiter distalwärts die Berührungen erfolgen. An den einzelnen Fingern ist die Lokalisationsfähigkeit nicht immer gleich. In den ersten Monaten nach der Verletzung erscheint der 4. und 5. Finger frei von der Störung, während die drei, vor allem an der Greifbewegung beteiligten Finger Daumen, Zeige- und Mittelfinger eine deutliche Störung der Lokalisation aufweisen. Später lässt sich dieser Unterschied hinsichtlich der einzelnen Finger nicht mehr mit solcher Bestimmtheit nachweisen. Die Lokalisationsstörung findet sich 
auch an den anderen Fingern. Es ist bemerkenswert, dass das Lokalisationsvermögen $\mathrm{zu}$ der einen Zeit wesentlich besser ist, als zur anderen, ohne dass die Ursache dafür klar läge. Fin Zusammenhang mit einer allgemeinen Bewusstseinsstörung nach überstandenen epileptischen Anfällen, woran bei dem.Kranken gedacht werden musste, bestand nicht; diese Nachwirkung liess sich gelegentlich auch feststellen, doch war dann die Störung allgemein und nicht nur auf eine Seite beschränkt.

Ein ähnliches Verhalten wie hinsichtlich der Lokalisationsfähigkeit fand sich beim Tasten. Während grosse Gegenstände, wie ein Stück Holz, Apfelsine, Bürste, Schuh, meist erkannt wurden, bestand eine deutliche Störung im Abtasten kleiner Gegenstände. Doch gab es auch hier Schwankungen. An einzelnen Tagen wurde besser taktil erkannt, als an anderen. In den Protokollen vom 9. II. 1901 und 31. III. 1903 scheint es, als ob eine Parallelität zwischem gutem Tasten und gutem Lokalisationsvermögen bestände. Es findet sich in dem ersteren eine verhältnismässig grosse Zahl von guten Reaktionen, im zweiten erkennt er, was ihm sonst nie gelungen war, das $\mathrm{R}$ durch Abtasten des Holzbuchstabens.

Bemerkenswert ist die Art der Fehlreaktionen. Vielfach beschreibt er die einzelnen taktilen Komponenten ganz leidlich, ohne zur Identifikation zu gelangen, während er zu anderen Zeiten denselben Gegenstand mit ganz anderen Eigenschaften belegt. Das eine Mal bezeichnet er die ihm in die Hand gegebene Schraube als "kalt, Eisen, oben wie ein Knopf", ein ander Mal als „dünn, weich, lang und schmal, Gummi oder Bleistift. Bei einem Kamm nennt er folgende Kriterien: „blechartig, wie Stück Blech, unten so zahnartig wie bei einer Säge"; bei einer späteren Prüfung: „Das ist Holz, so gross wie mein Zeigefinger". Einen Hemdknopf bezeichnet er einmal als "ganz klein, rund, vielleicht ein Ring", ein ander Mal als dreikantig, ein drittes Mal bemerkt er ihn überhaupt nicht. - Man sieht, es sind keineswegs gleichmässige und gleichbleibende Reaktionen, die durch denselben Reiz hervorgerufen werden. Als häufigste Ursache der Fehlreaktionen tritt das Symptom des Haftenbleibens hervor. Auf den Gegenstand, den er bei der Tastprüfung nicht erkannt hat und der ihm dann gezeigt worden ist, rät er in den folgenden Tastversuchen mit grosser Regelmässigkeit wieder, anch wenn es sich um etwas ganz Disparates handelt. So bält er einmal ein Zehnpfennigstück, ein ander Mal einen Kamm für einen Schlüssel, weil ihm der Schlüssel kurz zuvor gezeigt worden ist, und die eiserne Schraube erscheint ihm aus demselben Grunde als Radiergummi. Diese Erscheinung des Haftenbleibens ist eine bei Gehirnerkxankungen, namentlich bei kortikalen Affektionen, be- 
kannte Erscheinung, Ebenso wie bei den aphasischen Störungen erweist sich auch hier die Erscheinung für den Untersuchungsgang oft störend, insofern der Kranke von einer Detailbeschreibung dessen, was er fühlt, durch die perseverieirende optische Erinnerung des rorher gesehenen Gegenstandes abgelenkt wird.

Die Lage-, beziehungsweise Bewegungsempfindung weist bei der gewöhnlichen klinischen Untersuchungstechnik bei der Mehrzahl der Untersuchungen keine Störung auf. Nur einmal scheint er passive Bewegungen des Zeigefingers in einzelnen Gelenken schlechter wahrzunehmen. Tatsächlich sind aber doch die aus diesem Sinne hervorgegangenen Wahrnehmungen ungenau. Lässt man den Kranken bej geschlossenen Augen verschiedene Entfernungen vom linken Daumen und Zeigefingerkuppe abschätzen und auf den Zollstab übertragen, so macht er grobe Fehler und zwar schätzt er meist zu klein. Damit hängt es wohl zusammen, dass er auch die Grössenverhältnisse der Gegenstände sehr schlecht abschätzt.

Wir haben es also neben der Taststörung mit einer Störung in der Lokalisation der Berührungen und der talktilen Distanzwahrnehmung zu tun.

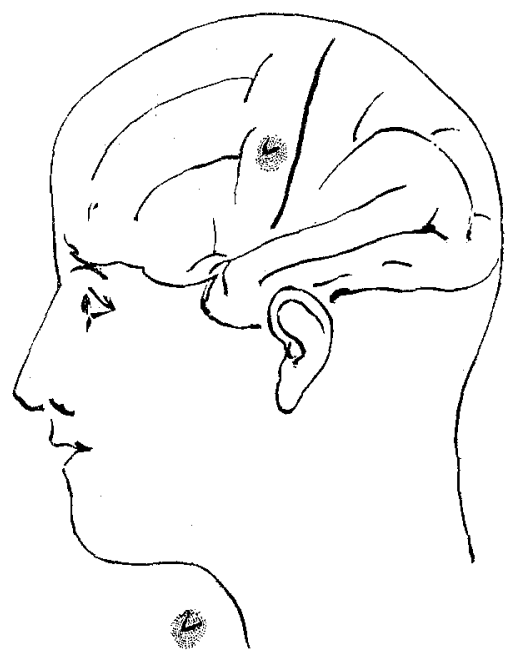

Mutmaßlicher Sitz der Läsion in den ersten 3 Fällen. (Schema v. A. Starr.)

\section{Beobachtung II.}

Schädelverletzung. Eingekeibtes Knochenstück in der Rinde der Zentralwindung. Parese und leichte sensible Störung der rechten Hand. Operative Entfernung des Knochenstucks, danach Verschlimmerung der Taststörung und der Lokalisationsfähigkeit.

J. B., 24jähriger Arbeiter. Auf dem linken Scheitelbein besteht eine tiefe Impression, welche die Folge eines vor Jahresfrist erhaltenen Hufschlages ist. Die Narbe liegt senkrecht zur Mittellinie des Schädels in einer direkt dem Ohransatz zustrebenden Linie, sie ist $8 \mathrm{~cm}$ lang. Von der Hautnarbe aus geht eine Knochendepression nach vorne. Etwa in der Mitte der Hautnarbe ist Pulsation zu fühlen. Der Befund von seiten des Nervensystems ist folgender: 
Es besteht eine Parese der rechten Hand. Die Finger stehen in der Ruhelage nicht in der normalen Parallelstellung. Bei gerader Stellung der Hand sind Mittel- und Zeigefinger etwas dorsal flektiert, Daumen und Zeigefinger sind in dem 1. Phalangealgelenk etwas gebeugt. Der Handschluss ist gut möglich und geschieht leidlich kräftig. B. klagt, er habe manchmal Spannungen in den Fingern. Die Opposition des Daumens ist sämtlichen Fingern gegenüber erschwert. Der rechte Adductor pollicis macht einen etwas atrophischen Eindruck, keine Störung der elektrischen Erregbarkeit.

Im Handgelenk besteht leichte Spannung, ebenso im Schulter- und Ellbogengelenk. Die Einzelbewegungen der Finger sind etwas verlangsamt. Bei Augenschluss tritt eine Ungeschicklichkeit der feineren Fingerbewegungen deutlicher hervor. Er hält die Schreibfeder zwischen Zeige- und Mittelfinger. Die Schrift ist an einzelnen Stellen ansfahrend, aber gut leserlich. Er benutzt die Hand. Kann den Rock zuknöpfen. Reflexsteigerung am Arm. - Bein und Facialis sind intakt.

Subjektiv gibt Pat. an, dass sich die Gegenstände rechts „zäher" anfüblen als links, auch habe er ein Kältegefühl in der rechten Hand. Die Berührungsempfindung ist gut. Die Reaktion ist gegenüber links verlangsamt. Der Schwellenwert für 2 getrennte gleichzeitige Berührungen beträgt rechts und links 2 mm an den Fingerkuppen. Die Lokalisation der Berührungen geschieht am Handteller und Handrücken gut, an den Fingern ist sie zunächst gut, nach mehrfacher Wiederholung wird sie schlechter. Passive Bewegungen werden in allen Fingergelenken wahrgenommen and auch ihrer Richtung entsprechend angegeben, nur am Ringfinger wird die Richtung nicht genau angegeben. Stellungen, welche der rechten Hand bei geschlossenen Augen gegeben werden, werden mit der anderen richtig nachgeahmt. Beim Abtasten von Gegenständen bei geschlossenen Augen erkennt er zunächst alles richtig, er braucht aber länger, nach kurzer Zeit - schon bei dem sechsten Gegenstand wurde die Tastleistung entschieden schlechter. Er erkennt dann ein Fünfmarlzstück, ein MetermaB, einen Schlüssel, ein Stück Schnur nicht mehr, während er links sofort identifiziert.

Operation (Geheimrat von Mikulicz): Exzision der Narbe. Nach hinten von der pulsierenden Stelle ist der Kuochen intakt, nach vorne zu findet sich eine grosse Depression. Mit Hammer und Meissel wird ein ungefähr $5 \mathrm{~cm}$ langes und $3 \mathrm{~cm}$ breites Knochenstück, das sich senkrecht in die Hirnsubstanz hineinerstreckt, entfernt. Es liegt dann eine komplizierte Wunde mit zerrissener Dura und freiliegender Rinde blos. Die Dura wird abpräpariert und durch Katgut vereinigt. Die Stelle, an der die Rinde lädiert war, entspricht der Mitte der vorderen Zentralwindung.

$\mathrm{Nach}$ der Operation ist die grobe Kraft zunächst rechts und links schwächer als vor der Operation. Schon am zweiten Tag ist dies ausgeglichen. 10 Tage nach der Operation ist die rechte Hand kräftiger als vorher. Die Spannung im Schulter- und Ellbogengelenk hat aufgehört. In motorischer Beziehung ist im übrigen an dem Befund der Hand nichts geändert.

Hinsichtlich der Sensibilitat ist eine Änderung eingetreten, insofern, als die Genauigkeit der Lokalisation der Berührungen wesentlich verschlechtert ist, während die Berührungsempfindung selbst er- 
Ialten bleibt. Hinsichtlich der Lageempfindung besteht derselbe Befund wie früher.

Die Tastfähigkeit ist deutlich verschlechtert. Er erkennt Schlüssel, Federhalter, Bleistift, Uhr und kleinere Gegenstände nicht.

Tastfähigkeit und Lokalisationsvermögen bessern sich bis zur Entlassung noch, bleiben aber beide gestört.

Als Folge einer traumatischen Rindenläsion besteht eine isolierte Parese der rechten Hand. Die feineren Fingerbewegungen sind gestört, aber nicht aufgehoben. Insbesondere ist die Beweglichkeit des Daumens geschädigt. Es besteht eine subjektive Sensibilitätsstörung. Objektiv ist vor allem die rasche Ermüdbarkeit des taktilen Erkennens auffällig. Eine grobe Störung der Bewegungsempfindung ist nur an einem Finger nachweisbar, auch das Lokalisationsvermögen ist gut, doch erschöpft sich auch dieses nach mehreren Versuchen sehr rasch.

Der Erfolg der Operation macht sich nach zwei Seiten hin bemerkbar. Fine leicht tonische Spannung, die in den Armen bestanden hat, löst sich; es hatte sich offenbar um einen von dem Fremdkörper ausgehenden Reizzustand gehandelt, der sich in einer dauernden Zunahme des Tonus in den Muskeln des Arms und in zeitweilig auftretenden Spannungen in den Fingern geäussert hatte. Bemerkenswerter Weise waren niemals Anfälle von Jacksonscher Epilepsie aufgetreten. Hinsichtlich der Sensibilität zeigte sich, wie schon in dem vorhergehenden Falle, eine Parallelität in dem Verhalten der Lokalisationsfähigkeit und des Tastvermögens. Beide wiesen als Folge der Operation eine deutliche Verschlechterung anf:

Erwähnenswert ist noch die beobachtete Atrophie im Bereiche der Daumenmuskulatur.

\section{Beobachtung III.}

Linksseitige Depression. Kortikale Anfälle. Leichte motorische Schwäche der Hand. Störung der Bewegungsempfindung, der Berührungsempfindung, des Lokalisationsvermögens, geringe Taststörung.

A. S., oberschlesischer $30 \mathrm{jähr}$. Arbeiter, ist vor einigen Jahren die Treppe herabgestürzt und hat sich dabei eine Wunde über dem linken Scheitelbein an der Grenze des Stirnbeins zugezogen. Nach dem Unfall war S. zunächst aphasisch und rechts gelähmt. Beides bildete sich nach kurzer Zeit (14 Tagen) zurück. In der Folgezeit hatte der Kranke zeitweise auftretende Anfälle, die mit Streckkrampf in der rechten Hand anfingen, daran schlossen sich Zuckungen in der rechten Gesichtsfeldhälfte; dann ging das Bewusstsein verloren. Gelegentlich traten auch isolierte Zuckungen in der Hand ohne Bewusstseinsverlust auf.

Es besteht eine breite Impression des linken Seitenwandbeins etwas Dentsche Zeitschr. f. Nervenheilkunde. XXVI. Bd. 
oberhalb des Schläfenbeins fast parallel der Sagittallinie zwischen zwei Senkrechten, die vom vorderen Ohrläppchenwinkel und vom hinteren äusseren Ohrrand auf die Sagittalnaht gezogen werden.

Es besteht eine geringe Herabsetzung der Kraft der rechten Hand. Die Fingerbewegungen sind rechts gut, bei offenen Augen ist der Gebrauch und die Beweglichkeit der Hand überhaupt kaum gestört. Die Opposition des Dawmens geschieht ohne Defekt, doch macht sich bei schnellen Fingerbewegungen eine Verlangsamung gegenüber der anderen Hand bemerklich. Die Schriftzüge zeigen keine grobe Störung, doch braucht er lange Zeit zum Schreiben. Bei geschlossenen Augen wird die Ataxie der feineren Fingerbewegungen deutlicher. Die Handhaltung in der Rube zeigt eine Störung, insofern die Finger nicht ganz in einer Ebene stehen. Keine Spasmen.

Im Gebiete des Mundfacialis ist nichts Pathologisches nachweisbar. Auch der weiche Gaumen hebt sich gleichmässig. Die Zunge wird gerade herausgestreckt. Schnelle Bewegungen nach rechts sind etwas beeinträchtigt. Die Sprache ist etwas stolpernd und wenig prononziert.

Am Bein besteht eine leichte Steigerung der Sehnenreflexe auf der rechten Seite, kein Klonus und kein Babinskisches Zehenphänomen. Mitunter scheint es, als ob eine geringe Schwäche der Dorsalflexion des Fusses and der Kniebeuger bestände. Der Gang zeigt keine Spur einer hemiplegischen Störung.

S. gibt an, er habe ein Kältegefühl in der rechten Hand, im übrigen sei die Empfindung sehr wechselnd. Dies bestätigt sich auch bei der Untersuchrong, insofern die Schmerzempfindung an der rechten Hand einmal abgestumpft erscheint, während sich eine Viertelstunde später keine Differenz ergiebt. Die Berührungsempfindung ist erhalten, doch sind die Angaben nicht ganz so scharf wie links. Zwei getrennte Berührungen mit dem Tasterzirkel werden an den Fingerkuppen rechts durchschnittlich etwa bei einem Abstand von 5-7 mm, links bei $3-4 \mathrm{~mm}$ wahrgenommen.

Die Lokalisation der Berührungen an der Hand geschieht ungenau, sie ist an den volaren Endgliedern besonders schlecht. Strichförmige Berührungen werden gut lokalisiert. Die Bewegungsempfindung ist am Handgelenk und den basalen Phalangen rechts und links gleich, an den Endgliedern ist sie rechts deutlich unsicherer als links.

Am Vorder- und Oberarm und am rechten Bein besteht keine Störung der Empfindlichkeit. Nur ist die rechte Fusssoble kitzliger als die linke.

Das Abtasten von Gegenständen mit der rechten Hand geschieht nicht schlecht. Es wird vieles erkannt, dagegen werden feinere Unterschiede an den Dingen nicht wahrgenommen. Ein Bleistift von eckiger Form wird von einem runden nicht als verschieden erkannt. Ebenso verhält es sich mit Schlüsseln von verschiedenen Grössen. Kleine Gegenstände werden öfters nicht erkannt.

Dass es sich auch hier um eine kortikale Läsion handelt, wird zunächst schon durch die Depression wahrscheinlich gemacht. Weiterhin spricht dafür die Art, wie die definitiven Herderscheinungen sich verteilen. Wir haben es zwar nicht mit einer ganz aussehliesslich die Armregion betreffenden Störung zu tun, da eine leichte Störung der 
Ziungenbeweglichkeit und eine Reflexsteigerung an der rechten unteren Extremität besteht. Die eigentlichen Ausfallserscheinungen betreffen aber lediglich die Hand. In motorischer Beziehung sind die feineren Abstufungen der Fingerbeweglichkeit geschädigt, jedoch ohne stärkere Beteiligung der Daumen- und Zeigefingerbeweglichkeit.

Hinsichtlich der Sensibilität sind deutliche Störungen der Berührungsempfindung, des Lokalisationsvermögens und der Bewegungsempfindung nachzuweisen. Die Störung der letzteren beiden Qualitäten beschränkt sich auf die Endglieder der Finger. Die Störung der Bewegungsempfindung ist hier wesentlich deutlicher, als in den zuvor genannten Fällen. Trotzdem ist die Störung des Tastens hier nicht erheblicher als bei den beiden vorgenannten Kranken. Sie entspricht, soweit dies überhaupt abschätzbar ist, in der Stärke ibrer Ausbildung dem Grade der vorliegenden Sensibilitätsstörung.

Überempfindlichkeit der Fusssohlen habe ich schon einmal in einem anderen Falle kortikaler Läsion beobachtet. ${ }^{1)}$ v. Monakow, Dejerine u. A berichten Ähnliches bei Fällen kortikaler Läsion im Bereiche der Zentralwindungen.

Beobachtung IV.

Depression des rechten Parietalbeins. Linksseitige Parese des Arms mit besonders starker Störung der Daumenbeweglichkeit.

Partielle Tastlähmung,

H., 33jähriger Arbeiter. In Jahre 1899 durch Sturz auf den Kopf Verletzung der rechten Schädelhälfte. Nach dem Unfall 14 Tage lang Krrämpfe. 3 Stunden nach dem Unfall konnte er trotz erhaltenen Bewusstseins nicht sprechen.

Eine mehr als talergrosse Depression im hinteren Teile des Scheitelbeins bis zur Schläfenschuppe reichend. Sie liegt im mittleren Drittel einer von der offinung des äusseren Ohres nach der Sagittalebene des Schädels gelegten Senkrechten.

Linke Nasenlippenfalte etwas verstrichen. Die Zunge zittert, wird gerade herausgestreckt. Ihre Bew eglichkeit ist nach links etwas beschränkt. Gaumensegel, Augenschluss von guter Funktion. Die linke Schulter steht eine Spur tiefer, auch beim Heben bleibt sie etwas zurück. Hebung der Arme geht beiderseits gleich hoch, links etwas langsamer. Die Streckung und Bengung des Oberarms geschieht links schwächer als rechts.

$H$. benützt die Hand beim Aus- u. Anziehen kaum. Auf Nötigung gelingt das Aufknöpfen des Roclss und ähnliches, jedoch unter Schwierigkeiten. Beide Hände zittern beim Ausstrecken. Beugung der Hand plantarwärts gut, dorsalwärts rechts weniger kräftig als links. Händedruck schwach. Pronation und Supination entsprechen dem physiologischen Verhältnis. Die Opposition des Daumens links sehr schlecht. Berührung der Kuppe des Ring- und Kleinfingers nicht möglich. Die feineren Bewegungen der Finger sind er-

1) Beiträge zur Hiruchirurgie und Hirnlokalisation. Monatsschrift für Psychiatrie und Neurologie. Bd, III. S. 298. 
schwert. Addultion der Finger ist schlecht, Spreizung gelingt besser. Feinere Greifbewegungen geschehen bei geschlossenen Augen besonders schlecht. Bei passiven Bewegungen der Finger keine Steifigkeit. Periostreflex rechts und links ohne Differenz. Bei Dorsalflexion der Finger bleiben sie nicht in einer Ebene, der Mittelfinger bleibt etwas zurück. Am Bein Reflexsteigerung links, kein Fussklonus, geringe Differenz in den Prädilektionsmuskeln. Keine Cirkumdnktion des Fusses beim Gang. Die Schmerzempfindung erscheint auf der ganzen linken Seite in geringem Maße herabgesetzt. Die Berührungsempfindung ist mit der nachbezeichneten Ausnahme erhalten, doch sind die Angaben oft unsicherer als rechts. Strichförmige Berührungen fühlt H. stets. Am Handteller und am 3., 4. u. 5. Finger ist auf der Dorsalund Volarseite die Berührungsempfindung herabgesetzt und die Lokalisation der Berührungen sehr ungenau. Bewegungen werden im Handgelenk gut, an den genannten 3 Fingern undentlich und bei kleinen Exkursionen in der Richtung unsicher wahrgenommen. Daumen und Zeigefinger zeigen aucb. leichte Lokalisationsstörung und Lageempfindungsstörung, sie verhalten sich aber hinsichtlich dieser Empfindungsqualitäten deutlich besser als die anderen Finger. Bei der Tastprüfung ist $\mathbf{H}$. infolge der geringen Beweglichkeit des Daumens behindert; zunächst scheint es, als ob eine vollständige Tastlähmung bestände. Bei genauerer Prüfung zeigt sich aber, dass er $\mathrm{z}$ wischen Zeigefinger und Daumen, wenn es auch lange dauert, bis er die Gegenstände hier richtig liegen hat, richtig identifiziert. Er erkennt Schlüssel, Papier, Bleistift, Uhr, Kette, Taler, Portemonnaie u.s.w. I_ässt man ihn dagegen zwischen den 3 leichter beweglichen Fingern abtasten, so erkennt er hier nichts.

Auch hier liegt keine ganz isolierte monoplegische Störung der oberen Extremität vor. Reflexsteigerung am linken. Bein, Erschwerung der Zungenbeweglichkeit nach links, ausserdem die Herabsetzung der Schmerzempfindung auf der ganzen linken Seite sprechen für eine ausgedehntere Schädigung. Dass es sich in der Hauptsache aber doch um eine kortikale Schädigung der Armregion handelt, wird einerseits durch die Art der Läsion wahrscheinlich gemacht, weiterhin spricht die vorwiegende Schädigung der feineren Fingerbewegungen und der Daumenbeweglichkeit in diesem Sinne.

Die Sensibilitätsverhältnisse sind eigenartig. Zunächst ist bemerkenswert, dass der Daumen sich hinsichtlich der Motilität wesentlich anders verhält, als hinsichtlich des Gefühls. Die Beweglichkeit ist erheblich gestört, während die Sensibilität fast intakt ist. Es liegt an der Hand eine partielle Tastlähmung vor, mit der einen Hälfte der Hand kann er taktil erkennen, mit der anderen nicht. Gleichzeitig liegt allerdings auch eine Differenz in den Sensibilitätsverbältnissen vor, indem an dem nicht tastfähigen Teil der Hand auch die Sensibilität eine etwas stärkere Störung aufweist. Auf die Beobachtung ist unten noch einzugehen. 


\section{Beobahtung V.}

Plötzlich aufgetretene Parese der Hand und aphasische Störung. Motorische Ungeschicklichkeit. Lokalisation der Berührung, Lageempfindung an den Fingern grob gestört. Tastlähmung. 4 Wochen später zweite Attacke mit anschliessender Parese des Beins.

Ingenieur G., 51 J. alt, hat vor 12 Jahren Lues gehabt. Im Juli 1902 jlötzlich während eines Badeaufenthalts eines Morgons grosse Schwierigkeit in der Sprache und im Wortfinden, und Schwäche der rechten Hand. Am Bein bemerkte der Kranke nichts; er ging sofort zum Arzte, der ihm riet nach Hause zu reisen. Hier lässt sich einige Tage nachher folgender Befund erheben: Geringe Schwäche im rechten Facialis. Die Sprache ist gestört, der Kranke hat grosse Schwierigkeit, wenn er etwas sagen will, das Wort zu finden. Die Aussprache ist beim Spontansprechen schwerfällig, abgesetzt, in einer Weise, wie sie bei motorischen Aphasien, die sich in der Rückbildung befinden, angetroffen wird. KeineStörung des Wortverständnisses. Nachsprechen im ganzen sehr gut, nur bei ganz schwierigen Worten etwas stolpernd. Leseverständnis für Einfaches gut. Zeitungsartikel gibt er an nicht zu verstehen. Die Schrift ist stark ataktisch; gelegentlich zeigen sich litteralparagraphische Elemente. Der Gang ist ungestört. Die Patellar- und Ichillessehnenreflexe ohne Differenz, kein Babinski. Grobe Kraft beider Beine ohne Unterschied. Die Sensibilität der Beine zeigt keinen Unterschied.

Am Arm besteht eine mässige Parese. Die Periostreflexe sind rechts gesteigert.

Feinere Bewegungen der Finger sind ungeschickt, bei geschlossenen Augen noch schlechter. Pat. war früher Geigenspieler, ist jetzt gänzlich ausser stande zu spielen. Die Opposition des Daumens gelingt, ist aber verlangsamt und erschwert. Die grobe Kraft des Händedrucks ist etwas herabgesetzt. Keine Kontrakturstellung. Die Berührungsempfindung ist an der linken Hand erhalten. Die Lokalisation ist an der Hand und am stärksten an den Fingern gestört. Am Vorderarm ist sie intakt. In ähnlicher Ausdehnung zeigt anch die Bewegungsempfindung eine Störung. Bewegungen im Handgelenk werden gefühlt und der Richtung entsprechend angegeben. Dagegen werden Bewegungen der Finger in allen Gelenken, besonders in den distalen so gut wie gar nicht gefühlt. Er weiss die Finger, die bewegt werden, nicht immer zu nennen. Stellungen der rechten Hand kann er mit der linken nicht nachahmen. Es besteht eine vollständige Tastlähmung auch für ganz grobe Dinge.

Pat. hat subjektiv an der rechten Hand ein taubes Gefühl und häufig Anfälle von Parästhesien, die anf Hand und einen Teil des Vorderarms beschränkt sind.

- Vier Wochen nach dem ersten Anfall leichte Schwächeanwandlung mit nachfolgender Parese des Beins und stärkerer Sprachstörung. Es bildet sich nun auch eine typische Parese des Beins mit Spasmen aus. Der Befund am Arm bleibt derselbe.

In motorischer Beziehung tritt mit der Zeit unter Jodbehandlung eine Besserung ein. Der sensible Befund an der Hand mit starker Störung der Bewegungsempfindung und des Lokalisationsvèrmögens, ebenso die Tastlähmung 
bleiben unverändert. Hemianopsie war zu keiner Zeit nachweisbar. Der Augenhintergrund ist frei. Die Pupillen sind myotisch, reagiren aber gut anf Lichteinfall. Es besteht Unfähigkeit zur Konvergenz. - Pat. hat anschliessend an den Tod seiner Wirtschafterin, die an Carcinom gestorben war, wochenlang die Idee, er leide an Magenearcinom und konsultierte zahlreiche Ärzte. Später wurde diese Idee anschliessend an mehrfache Augenuntersuchungen von dem Gedanken, dass sich die Pupillen verändert hätten, viereckig geworden seien, abgelöst.

Seit einem halben Jahr ist der Kranke frei von hypochondrischen Ideen. Intelligenz, Aufmerksamkeit und Gedächtnis ist nicht gestört. Es besteht aber eine leichte emotionelle Erregbarkeit.

Dass es sich bei $G$. nach dem ersten Insult um eine kortikale, dem Kortex jedenfalls sehr nahe sitzende und ihn mitbeteiligende Affektion handelte, ist nach dem zunächst monoplegischen Charakter der Extremitätenbeteiligung und der von Anfang an auf die Hand beschränkten sensiblen Parese mit Bestimmtheit anzunehmen. Ungewöhnlich und nach dem Schema der sogenannten typischen Aphasien schwer zu rubrizieren ist die bei $G$. bestehende Sprachstörung. In formaler Beziehung entspricht die Sprechweise der eines Kranken, den ich anderwärts ${ }^{1}$ ) beschrieben habe und bei dem eine artefakte durch Pialäsion verursachte kortikale Läsion des Fusses der 2. linken Stirnwindung vorgelegen hatte. Die einzelnen Worte werden unter sichtbarer Anstrengung langsam mit monotoner, gleichmässiger Accentuierung ausgesprochen. Eine artikulatorische Sprachstörung besteht nicht. In der Spontansprache ist das Wortfinden ausserordentlich erschwert und der Kranke kommt dadurch leicht in ärgerliche Erregung. Nachsprechen gelingt gut. Das Benennen vorgelegter Gegenstände bietet keine Schwierigkeiten und auch hierin. verhält er sich wie Kranke mit leichter Schädigung in der Gegend der Brocaschen Windung. Auch die leichte Störung des Leseverständnisses würde bei Läsionen dieser Gegend nichts Ungewöhnliches sein. Jedenfalls spricht auch die Art der bei $G$. vorliegenden Sprachstörung für eine kortikale Affektion.

Hinsichtlich der Sensibilität unterscheidet sich der Fall von den bisherigen dadurch, dass eine besonders starke Störung der Bewegungsempfindung vorlag und dass die Tastlähmung vollständig ist und bleibt.

In allen Fällen ist die Diagnose einer überwiegend kortikalen Läsion wahrscheinlich. Was den speziellen Sitz der Herde anlangt, so kann man in den beiden ersten. Fällen mit ziemlicher Bestimmtheit etwa die Mitte der vorderen Zentralwindung als

1) Zur Kenntnis der Rückbildung motorischer Aphasien. Mitteilungen aus den Grenzgebieten etc. von Mikulicz u. Naunyn. X. Bd. 1. Heft. 
Ort der hauptsächlichsten Verletzung bezeichnen. Nimmt man im dritten Falle an, dass die Stelle der tiefsten Depression dem Orte der Gehirnverletzung entspricht, so kommt man auch in diesem Falle auf dieselbe Gegend. Die eigentümliche Art der Sprachstörung, welche hier und bei der Mehrzahl der hierher gehörigen linksherdigen Fälle der Literatur angetroffen wird, steht der motorischen Aphasie näber als der sensorischen. Man trifft sie, wie schon ausgeführt, bei Läsionen, die den Fuss der zweiten linken Stirnwindung mitbetreffen. Es lässt also auch dies Moment eine stärkere Beteiligung der vorderen Zentralwindungspartien annehmen. Wernicke glaubt, dass überwiegend die Mitte der hinteren Zentralwindung Sitz der Läsion bei kortikaler Tastlähmung sei, während v. Monakow im ersten Wernickeschen Falle den Herd mehr nach vorne, also auch in die vordere Zentralwindung lokalisiert (vergleiche die Abbildung in v. Monakows Gehirnpathologie S. 411).

Was die Motilität bei kortikalen Läsionen anlangt, so ergeben unsere Fälle nichts Neues. Grobe Bewegungsausfälle pflegen nicht vorzuliegen. Am schwersten geschädigt sind die feineren motorischen Fertigkeiten. So ist dem Kranken R. das Klavierspiel, $G$. das Geigen unmöglich geworden. Die Schrift ist entweder nicht, oder im Sinne einer leichten Ataxie verändert. Nur bei G. macht sich gelegentlich eine litterale Paragraphie bemerkbar, die mit der bestehenden aphasischen Störung in Verbindung zu bringen ist. Eine stärkere Daumenparese, die von manchen Autoren als charakteristisch für kortikale Läsionen angesehen wird, fand sich in 3 Fällen. Eine Herabsetzung der motorischen Kraftleistung der Hand ist in der Mehrzahl der Fälle nachweisbar, notwendig ist sie nicht, so fehlt sie im Falle I.

Für das Verhalten der Sensibilität bei kortikalen Läsionen ergeben sich aus unseren Beobachtungen folgende Punkte:

Die Sensibilitätsstörungen sind am ausgesprochensten in den distalen Partieen der Extremitäten. Am stärksten sind stets die Endglieder der Finger beteiligt, proximalwärts schwächt sich die Gefühlsstörung ab. Eine den Gliedabschnitten entsprechende Verteilung der Sensibilität findet sich nur in diesem Sinne. Für eine eigentliche Projektion der Sensibilität in der Rinde nach Gliedabschnitten im Munkschen Sinne geben unsere Beobachtungen keine Anhaltspunkte. Weder in unséren Fällen, noch auch sonst in der Literatur, soweit sie mir bekannt, finden sich Beobachtungen, in denen als definitives Ausfallssymptom etwa nur die oberen Gelenkabschnitte sensibel geschädigt wären, wäbrend die Hand frei bliebe oder auch nur eine geringere Schädigung aufwiese. 
Wir sahen, dass einzelne Finger stärker an der sensiblen. Störung teilnahmen als andere. Eine gliedweise Übereinstimmung von motorischer und sensibler Parese besteht nicht. So besteht im Falle IV am Daumen eine starke Störung der Motilität, während er in sensibler Beziehung so gut wie intakt ist.

Betrachtet man die einzelnen sensiblen Qualitäten bei unseren Rindenläsionen, so zeigt sich eine gewisse Mannigfaltigkeit der Befunde. Für die Schmerz- und Berührungsempfindung ergibt sich, dass sie gar nicht oder nur in ganz geringem Grade gestört sind. Es scheint nach unseren Fällen, dass sie dann gestört sind, wenn eine grössere Ausdehnung des Herdes eine leichte kortikale oder subkortikale Mitschädigung benachbarter Territorien mit sich bringt. Dass eine Schädigung der Rinde der Armregion ohne Störung der Schmerz- und Berührungsempfindung vorhanden sein kann, beweisen die Kranken R. und G., weiterhin von neueren Beobachtungen der erste Fall Wernickes und eine Beobachtung Redlichs, bei der es sich um eine operative Entfernung der Handregion gehandelt hatte. Ob es deshalb nötig ist, die kortikale Endstätte für die Berührungsempfindung anderswohin, z. B. in das Parietalhirn zu lokalisieren, kann dahin gestellt bleiben. Nach den Beobachtungen bei der Rückbildung frischer Rindenläsionen steht jedenfalls fest, dass sich Störungen dieser Qualität rasch zurückbilden. Ich kann dabei auf eine eigene Beobachtung verweisen, bei der es sich um die operative Entfernung eines grossen Teils der Zentralwindung und des angrenzenden Stirnhirn wegen eines Tumorhandelte. ${ }^{1}$ ) Es bestand hier nach der Operation ein Verlust der Berührungsempfindung; der sich auf Hand, Arm und Rumpf linkerseits erstreckte. Zwei Tage nach der Operation war sie schon zum Teil zurückgekehrt und 6 Tage nach der Operation bestand lediglich noch an den Fingern und zwar vor allem an den Endgliedern eine Herabsetzung der Berührungsempfindung, die sich schliesslich bis auf die Fingerkuppen zuriickzog. Anders verhielt sich die Lageempfindung. Sie änderte ihren Charakter sehr viel weniger, wenn auch der zunächst absolute Ausfall am Arm sich insofern besserte, als Bewegungen in den grossen Gelenken später wieder wahrgenommen wurden. Es blieb aber eine grobe Störung der Lageempfindung am Arm zurück, die wiederum ihre stärkste A usbildung an den Fingern behielt. Es lassen sich aus dieser Beobachtung in gehirntopographischer Beziehung keine zwingendenSchlüsse für die Lokalisation der Berührungsempfindung ziehen, weil sich nicht entscheiden lässt, ob es sich bei der Störung der Berührungsempfindung vielleicht um Fernwirkung von seiten des Parietal-

1) Beiträge zur Hirnchirurgie und Hirnlokalisation. Monatsschrift f. Psychiatrie u. Neurologie. Bd. IIr. Heft IV. S. 297. 
hirns gehandelt hat, oder ob bei partieller Schädigung der Centralwindungen durch rasch vikariierendes Eintreten anderer Territorien Störungen der Berührungsempfindung ausgeglichen werden können. Jedenfalls aber scheint die Lage- und Bewegungsempfindung enger an die Sphäre der Zentralwindungen gebunden zu sein, als die Berührungsempfindung. Dafür sprechen auch unsere eben beschriebenen Fälle.

Eine besondere Besprechung erfordert das Lokalisationsvermögen und die Störung im Wiedererkennen von Gegenständen. Wir haben in allen Fällen eine Störung des Lokalisationsvermögens, d. h. die Fähigkeit, den Ort, an dem eine mittelstarke Berührung stattgefunden hat, ist gestört. Sie ist aber nie ganz aufgehoben. Es kommt in keinem unserer Fälle vor, dass der Kranke, wenn er die Berührung wahrgenommen hat, nicht wenigstens die berührte Extremität zu bezeichnen wüsste. Auch die stärkste Störung, die angetroffen wurde, ging nicht so weit, dass Berührungen der Finger an den Vorderarm lokalisiert wurden. Häufig ist die Störung derart, dass Fingerberührungen in den Handteller lokalisiert werden und dass überhaupt proximalwärts lokalisiert wird. Bei leichteren Graden werden zwar falsche Finger als berührt angezeigt, während die Querebene, in welcher die Berührung stattgehabt hat, richtig wahrgenommen wird. Eine Reihe von rasch folgenden punktförmigen Berührungen oder einzelne Striche werden in ihrer Richtung meist gut lokalisiert, auch wenn im übrigen eine starke Störung der Lokalisation vorzuliegen scheint. Starke druckförmige Berührung wird schärfer lokalisiert als mässig starke. Die Lokalisationsstörung zeigt starke Intensitätsschwankungen, sie ist im besonderen bei Wiederholung der Prüfung durch die Ermüdung beeinflussbar, und sie ist an verschiedenen Tagen verschieden.

In den drei letzten Fällen geht neben der Lokalisationsstörung eine deutliche Herabsetzung der Lage- und Bewegungsempfindung einher. In der Beobachtung II waren diese Qualitäten auch, aber nur ganz leicht alteriert. Bei dem kranken R. war die Empfindung für passive Bewegungen nur zeitweise und nur in einem ganz kleinen Bereiche gestört. Trotzdem war eine deutliche Störung in der feineren taktilen Aloschätzung von Distanzen linkerseits nachzuweisen. Die Lokalisationsstörung war in den Fällen mit ausgesprochener Störung der Bewegungsempfindung nicht stärker, als in der Beobachtung I und II. Förster ${ }^{1}$, der einen mit den letzteren 2 Beobachtungen übereinstimmenden Fall beobachtet hat, stützt damit seine Annahme, dass das Lokalisationsvermögen ein kortikaler Assoziations-

1) Über das Lokalisationsvermögen. Monatsschrift für Psychiatrie u. Neurologie. Bd. IX. S. 42.1901. 
vorgang sei, bei dem es sich im wesentlichen um Verknüpfung von. Berührungs- und Bewegungsempfindung handle. Wäre also die Berührungsempfindung einerseits und die Bewegungsempindung andererseits erhalten, so wäre eine bestehende Lokalisationsstörung als spezifisch assoziativ und zwar kortikal assoziativ zu betrachten. In wie weit diese Schlussfolgerung klinisch zwingend ist, kann an unserem Material nicht entschieden werden. Wichtig ist jedenfalls, dass allen unseren Fällen eine Störung des Lokalisationsvermögens und des taktilen Wiedererkennens gemeinsam ist. Der Fall II gab mir zuerst Veranlassung, auf das Zusammenvorkommen dieser beiden Erscheinungen zu achten, indem hier eine bemerkenswerte Parallelität in der Intensität der beiden Störungen gleich nach der Entfernung des Knochensplitters aus der Rinde zutage trat. ${ }^{1}$ ) Dass auch der Kranke R. gelegentlich in der Stärke der Lokalisations- und der Taststörung parallelgehende Schwankungen zeigte, ist erwähnt worden.

Die Fähigkeit, Gegenstände durch Tasten wiederzuerkennen, war in allen Fällen, wenn auch in verschiedenem Grade geschädigt. Ein völligex Ausfall dieses Vermögens bestand nur in dem letzteren Falle. Auf die besondere Stellung, die der Kranke H. (Beobachtung IV) einnimmt, wird noch hinzuweisen sein. Der einzige Fall, in welchem die Tastlähmung vollständig war, zeigte gleichzeitig die stärkste Schädigung der Lageempfindung. Sehr erheblich ist aber auch die. Störung des taktilen Wiedererkennens in den Fällen I und II, in denen die Lageempfindungsstörung kaum geschädigt ist. Bei dem Kranken S. ist die Störung des Wiedererkennens gering, die Störung der Lageempfindung deutlich.

Es haben sich früher eine ganze Reihe von Untersuchern schon bemüht, die Bedeutung der einzelnen Empfindungsqualitäten für die Stereognose festzustellen. Die Resultate waren sehr verschieden. Darüber, dass es sich nicht um die Funktion eines eigenen Sinnes, des stereognostischen Sinnes, sondern um einen komplizierten Erwerb handelt, dürfte jetzt Einigkeit bestehen. Unter den einzelnen Sinven hat Hoffmann dem Druck-, Raum- und Ortssinn für die Stereognose eine besondere Bedeutung beigelegt, während ihm der Bewegungssinn hierfür weniger wichtig schien. Redlich hält dagegen letzteren für sehr wesentlich und findet die stärkste Störung der Tastfähigkeit bei Mitbeteiligung der Hautsensibilität. Nach unseren Fällen könnte es scheinen, und ich habe früher diese Auffassung gehabt, dass die Fähigkeit, durch Tasten wiederzuerkennen, in einem Abhängigkeitsverhältnis

1) Der Fall ist erwähnt worden in der Sitzung des Vereins ostdeutscher Irrenärzte. Februar 1900. Zeitschr. f. Psychiatrie. V. Bd. S. 414. 
vom Lokalisationsvermögen stehe, insofern die taktile Grössenabschätzung von dem Lokalisationsvermögen beeinflusst wird. Indessen haben mich weitere Beobachtungen bei peripheren Läsionen darüber belehrt, dass stärkere Lokalisationsstörungen vorkommen, ohne dass das Erkennen durch den Tastsinn geschädigt ist. Es scheint vielmehr, dass es sich bei dem Zusammenvorkommen von Störungen des taktilen Wiedererkennens und des Lokalisationsvermögens bei kortikalen Läsionen um koordinierte Erscheinungen handelt.

Für die vorliegende Betrachtung handelt es sich nicht um die Frage, welchen Einzelsinnen beim Erwerb der taktilen Komponenten einer Gegenstandsvorstellung die grösste Bedeutung zukommt, sondern es kann nur zur Beantwortung der Frage mit beigetragen werden, wovon der Verlust des taktilen Wiedererkennens bei kortikalen Läsionen abhängig ist, ob speziell die von Wernicke behauptete Selbständigkeit der Tastlähmung gegenüber allen Einzelempfindungsqualitäten zu Recht besteht. Tatsächlich zeigen unsere beiden ersten Fälle das als charakteristisch für die Tastlähmung hervorgehobene Moment, eine starke Störung der Stereognose bei geringer, die Tastläbmung nach den Erfahrungen bei peripheren und spinalen Läsionen nicht erklärender; sensiblex Störung. Der Schluss, den Wernicke daraus gezogen hat, ist bekanntlich der, dass in der Zentralwindungsrinde die Assoziationskomplexe liegen, welche zur Bildung der taktilen. Komponente der Gegenstandsvorstellung erforderlich sind. Die Tast]ähmung beruhe auf einer Läsion dieser Assoziationskomplexe. Tatsächlich zeigt die kortikale Tastlähmung - auch abgesehen von der von Wernicke entdeckten relativen Selbständigkeit der Tastlähmung gegenüber den einzelnen Empfindungsqualitäten - klinisch die allgemeinen Symptome einer assoziativen Störung. Als solche möchte ich zunächst die Schwankungen in der Intensität der Ausfallserscheinung nennen im Vergleich zu dem wesentlich konstanteren Charakter, den die Ausfallserscheinungen von seiten des Projektionssystems zeigen, man denke z. B. an das unveränderliche Verhalten einer Hemianopsie im Vergleiche zu den Schwankungen des klinischen Bildes bei Seelenblindheit oder sensorischer Aphasie. Weiterhị ist das für die Läsion von Assoziationsbahnen charakteristische Symptom des Haftenbleibens bei den Fällen von kortikaler Tastlähmung stets nachzuweisen. Man kann darin sogar, wie ich nach den Fällen, die ich in letzter Zeit gesehen habe, annehmen muss, ein differentiell-diagnostisches Moment gegenüber den peripherisch bedingten Störungen im taktilen Wiedererkennen erblicken.

Entspricht nun der taktilen Komponente der Gegenstandsvorstellungen ein einheitlich zusammengesetzter, den elementaren Sinnesempfindungsendstätten übergeordneter anatomischer Assoziationskom- 
plex? Diese Frage muss im Hinblick auf unsere 4. Beobachtung aufgeworfen werden. Wir haben hier eine im eigentlichen Sinne des Wortes partielle Tastlähmung. An einem Teil der Hand (Zeigefinger und Daumen) gelingt es die richtige Gegenstandsvorstellung wachzurufen, während dies an dem anderen nicht möglich ist. Handelt es sich hier um eine kortikale Tastlähmung, so muss diese Beobachtung gegen ein einheitliches Rindenzentrum sprechen. Denn bei Läsion eines solchen müsste die Wachrufung der taktilen Gegenstandsvorstellung gleichmässig geschädigt sein, gleichgültig, ob die zentripetale Erregung von Zeigefinger und Daumen, oder ob sie von den 3 übrigen Fingern und dem Handteller ausgeht. Man müsste vielmehr zu der Auffassung kommen, dass es sich innerhalb der motorischen Region nicht um eine universelle assoziative Zusammenfassung, sondern um einzelne, ihrerseits selbständige, die Gegenstandsvorstellung vermittelnde taktile Assoziationskomplexe handelt, die noch in einer engen räumlichen Beziehung zu den kortikalen Endstätten der Perzeptionsorgane, d. h. der Finger ständen. An diese Möglichkeit muss gedacht werden. Vollbeweisend für ein solches Verhalten ist der Fall allerdings nicht, denn man kann einwenden, dass an den im taktilen Wiedererkennen geschädigten Fingern auch gleichzeitig die Sensibilität stärker geschädigt ist, als am Daumen und Zeigefinger - wenn auch freilich nicht so erheblich, dass damit allein schon die gänzliche Tastlähmung erklärt wäre. Immerhin ist diese Sensibilitätsstörung deshalb vielleicht höher einzuschätzen, weil sie an denjenigen Fingern besteht, die für den Tastakt normaler Weise weniger in Betracht kommen, als die spezifischen Tastorgane Zeigefinger und Daumen. ${ }^{1}$ )

In den Fällen III und $V$ tritt trotz kortikaler Läsion die von Wernicke betonte charakteristische Differenz zwischen Tastlähmung und erhaltener Sensibilität nicht herror. Die reinen Fälle sind eben offenbar nicht häufig. Es muss, wie es scheint, eine besonders günstige Konstellation vorliegen, wenn ganz überwiegend das Lokalisationsvermögen und das taktile Wiedererkennen betroffen sein soll. Vielleicht trifft dies dann vor allem zu, wenn der Herd überwiegend die Rinde der vorderen Zentralwindung erfasst, während schon ein geringes Tiefer- und nach Hintengreifen des Herdes sofort stärkere Lage- und Bewegungsempfindungsstörungen nach sich zieht (vergleiche hierzu den zitierten Fall v. Monakows) und das Bild der sogenannten reinen Tastlähmung stört. Immerhin bedarf für die klinische Diagnosenstellung die Dar-

1) Beim normalen Menschen ist übrigens nach Versuchen, die ich gemacht habe, die Geschwindigkeit, mit der Gegenstände zwischen Mittel-, Ringund Kleinfinger einerseits und Handteller andererseits erkannt werden, kàum geringer als zwischen Daumen und Zeigefinger. 
stellung Dejerines, dass die Differentialdiagnose zwischen kortikaler und Kapselhemianästhesie nicht durch den Charakter der Hemianästhesie, sondern nur durch Hilfsmomente anderer Art zu stellen sei, einer Korrekłur. Eine Störung des Lokalisationsvermögens und des taktilen Wiedererkennens bei im übrigen nur geringfügiger Sensibilitätsstörung: kann als charakteristisch für eine Rindenaffektion gelten.

Es mag zum Schlusse noch die Frage nach dem Erfolge eines operativen Hingriffs bei diesen kortikalen Herden berührt werden. In unseren Fällen handelte es sich mit Ausnahme des letzten um Depressionsfrakturen. Die Beseitigung einer Depression ist bei dem jetzigen Stande der aseptischen Technik, wenn lokale Druckempfindlichkeit und cerebrale Herdsymptome vorliegen, wohl immer indiziert. $\mathrm{Ob}$ es dagegen zweckmässig ist, eine in der Rinde selbst vorgefundene Narbe zu exzidieren, wenn bloss die lokalen Ausfallssymptome: Ungeschicklichkeit der feineren Fingerbewegungen, Tast- und Lokalisationsstörung eventuell mit einer gewissen sensiblen Schwäche vorliegen, erscheint mir zweifelhaft. Eine Wiederherstellung der Funktion ist bei diesen das feinere assoziative Gefüge der motorischen Rinde betreffenden Läsionen nach den bisherigen Erfahrungen offenbar nicht. möglich. Man kann aber durch die Operation die Ausfallserscheinungen verstärken. Anders liegt es natürlich, sobald kortikale Krämpfe oder dauernde tonische Spasmen vorliegen. Man wird dann durch einen operativen Eingriff, wie z. B. in unserem 2. Falle, die Gebrauchsfähigkeit heben können. 